Bull. Austral. Math. Soc.

06А06, 47н09, 47H10,54H25

VOL. 65 (2002) [105-107]

\title{
ANOTHER PROOF OF THE BROWDER-GÖHDE-KIRK THEOREM VIA ORDERING ARGUMENT
}

\author{
JACEK JACHYMSKI
}

\begin{abstract}
Using the Zermelo Principle, we establish a common fixed point theorem for two progressive mappings on a partially ordered set. This result yields the BrowderGöhde-Kirk fixed point theorem for nonexpansive mappings.
\end{abstract}

Assume that $K$ is a nonempty, closed and convex subset of a linear topological space and $f$ is a selfmap of $K$. Our purpose is to indicate a possibility of solving a problem of the existence of fixed points of $f$ with a help of the following result which is known in a literature as the Zermelo fixed point theorem (see Dunford and Schwartz [2, p. 5]). Its formulation given below is due to Bourbaki [1], who proved this theorem using Zermelo's ([7]) ideas of the proof of the well-ordering principle.

THEOREM 1. (Zermelo) Let $(P, \preceq)$ be a partially ordered set in which every chain has a supremum. Assume that $F: P \rightarrow P$ is progressive, that is, $p \preceq F p$ for all $p \in P$. Then $F$ has a fixed point. Moreover, given $p \in P$, an element

$$
H p:=\sup \bigcap\{A \subseteq P: p \in A, f(A) \subseteq A \text { and for every chain } C \subseteq A, \sup C \in A\}
$$

is well-defined, $H p$ is a fixed point of $F$ and $p \preceq H p$.

Let us define the family $\mathcal{M}_{f}$ and the operator $\mathcal{F}$ by

$$
\begin{aligned}
\mathcal{M}_{f} & :=\{L \subseteq K: L \neq \emptyset, L=\mathrm{cl}(\operatorname{conv} L) \text { and } f(L) \subseteq L\} \\
\mathcal{F}(L) & :=\operatorname{cl}(\operatorname{conv} f(L)) \text { for } L \subseteq K,
\end{aligned}
$$

where cl stands for the closure operator and conv $L$ is the convex hull of $L$. (Note $\mathcal{M}_{f}$ is nonempty since $K \in \mathcal{M}_{f}$.) Then it is obvious that $f$ has a fixed point if and only if $\mathcal{F}$ has a singleton as its fixed point. It is easily seen that $\mathcal{F}\left(\mathcal{M}_{f}\right) \subseteq \mathcal{M}_{f}$ and $\mathcal{F}(L) \subseteq L$ for $L \in \mathcal{M}_{f}$. If, furthermore, $K$ is compact, then every chain in a partially ordered set $\left(\mathcal{M}_{f}, \supseteq\right)$ has a supremum-the intersection of all its members. Since $\mathcal{F}$ is progressive on $\left(\mathcal{M}_{f}, \supseteq\right)$, Zermelo's theorem yields the following result (see also Kirk [6]).

Received 29th May, 2001

Copyright Clearance Centre, Inc. Serial-fee code: 0004-9727/02 \$A2.00+0.00. 
Proposition 1. Let $K$ be a nonempty, compact, convex subset of a linear topological space and $f$ be a selfmap of $K$ (not necessarily continuous). Then there exists a nonempty, compact, convex set $K_{*} \subseteq K$ such that

$$
\operatorname{cl}\left(\operatorname{conv} f\left(K_{*}\right)\right)=K_{*}
$$

that is, $K_{*}$ is a fixed point of $\mathcal{F}$.

However, we are more interested in the following question. When does the family Fix $\mathcal{F}$ of all fixed points of $\mathcal{F}$ contain a singleton? In particular, this is the case if $\mathcal{F}$ has a common fixed point with an operator having the property that every fixed point of it is a singleton. The following common fixed point theorem may be helpful here.

THEOREM 2. Let $(P, \preceq)$ be a partially ordered set and $P_{0} \subseteq P$ be nonempty and such that every chain in $P_{0}$ has a supremum in $P_{0}$. Let $F: P_{0} \rightarrow P_{0}$ and $G: P \rightarrow P$ be progressive mappings. If

$$
G(\text { Fix } F) \subseteq P_{0},
$$

then $F$ and $G$ have a common fixed point.

Proof: By Theorem 1, there exists a progressive mapping $H: P_{0} \rightarrow$ Fix $F$. Then the mapping $G \circ H$ is also progressive and

$$
(G \circ H)\left(P_{0}\right) \subseteq G(\text { Fix } F) \subseteq P_{0}
$$

By Theorem 1, $G \circ H$ has a fixed point $p_{*}$. Then

$$
p_{*} \preceq H p_{*} \preceq G\left(H p_{*}\right)=p_{*}
$$

which gives $p_{*}=H p_{*}$. Hence

$$
G p_{*}=G\left(H p_{*}\right)=p_{*}
$$

so $p_{*}$ is fixed under $G$. (Actually, $\operatorname{Fix}(G \circ H)=\operatorname{Fix} G \cap \operatorname{Fix} H$ for any progressive mappings $G$ and $H$.) Since $F \circ H=H, p_{*}$ is a fixed point of $F \circ H$. Repeating the above argument yields $p_{*}=F p_{*}$. Thus $p_{*}$ is a common fixed point of $F$ and $G$.

As an application, we shall give another proof of the Browder-Göhde-Kirk theorem-in the version of Kirk [5]-which is a fundamental result in the theory of nonexpansive mappings. We refer the reader to Goebel and Kirk [4, Chapter 4] for the terminology used below.

THEOREM 3. (Kirk) Let $K$ be a nonempty, weakly compact, convex subset of a Banach space and assume $K$ has normal structure. Then every nonexpansive mapping $f: K \rightarrow K$ has a fixed point.

Proof: We apply Theorem 2 taking the family of all nonempty, closed and convex subsets of $K$ for $P$ and setting (see (1)) $P_{0}:=\mathcal{M}_{f}, F:=\mathcal{F}$ and $\preceq:=\supseteq$. Further, $G$ 
is the Chebyshev operator assigning to each set of $P$ its Chebyshev centre. It is easily seen that $G$ is a selfmap of $P$ (see $[4$, p. 38]). Clearly, $G$ is $\supseteq$-progressive. Moreover, if $L \in \mathcal{M}_{f}$ and $L=\mathrm{cl}$ (conv $f(L)$ ), then the nonexpansivity of $f$ implies that $G(L)$ is $f$-invariant (see [4, proof of Theorem 4.1]), that is, the condition $G(\operatorname{Fix} F) \subseteq P_{0}$ holds. By Theorem 2, $F$ and $G$ have a common fixed point $K_{*} \in \mathcal{M}_{f}$. Since $K$ has normal structure, the equality $K_{*}=G\left(K_{*}\right)$ implies that $K_{*}$ is a singleton and thus $f$ has a fixed point.

Finally we emphasise that Fuchssteiner [3] was the first person who used Zermelo's theorem in the context of nonexpansive mappings. However, our approach seems to be quicker and more direct. Both these proofs are independent of the Axiom of Choice, whereas Kirk's [5] original proof relies on Zorn's Lemma.

\section{REFERENCES}

[1] N. Bourbaki, 'Sur le théorème de Zorn', Arch. Math. 2 (1949-50), 434-437.

[2] N. Dunford and J. Schwartz, Linear operators I: general theory (Wiley Interscience, New York, 1957).

[3] B. Fuchssteiner, 'Iterations and fixpoints', Pacific J. Math. 68 (1977), 73-80.

[4] K. Goebel and W.A. Kirk, Topics in metric fixed point theory, Cambridge Studies in Advanced Mathematics 28 (Cambridge University Press, Cambridge, 1990).

[5] W.A. Kirk, 'A fixed point theorem for mappings which do not increase distance', Amer. Math. Monthly 72 (1965), 1004-1006.

[6] W.A. Kirk, 'Nonexpansive mappings in metric and Banach spaces', Rend. Sem. Mat. Fis. Milano 51 (1981), 133-144.

[7] E. Zermelo, 'Neuer Beweis für die Möglichkeit einer Wohlordnung', Math. Ann. 65 (1908), 107-128.

Institute of Mathematics

Technical University of Lódź

Żwirki 36

90-924 Lódź

Poland

e-mail: jachym@ck-sg.p.lodz.pl 